\title{
Predictive Control for Distributed Smart Street Light Network
}

\author{
Pei Zhen Lee ${ }^{1}$, Sei Ping Lau ${ }^{2}$, Chong Eng Tan ${ }^{3}$ \\ Faculty of Computer Science and Information Technology \\ University Malaysia Sarawak (UNIMAS) \\ 94300, Kota Samarahan, Sarawak \\ Malaysia
}

\begin{abstract}
With the advent of smart city that embedded with smart technology, namely, smart streetlight, in urban development, the quality of living for citizens has been vastly improved. TALiSMaN is one of the promising smart streetlight schemes to date, however, it possesses certain limitation that led to network congestion and packet dropped during peak road traffic periods. Traffic prediction is vital in network management, especially for real-time decision-making and latency-sensitive application. With that in mind, this paper analyses three real-time short-term traffic prediction models, specifically simple moving average, exponential moving average and weighted moving average to be embedded onto TALiSMaN, that aim to ease network congestion. Additionally, the paper proposes traffic categorisation and packet propagation control mechanism that uses historical road traffic data to manage the network from overload. In this paper, we evaluate the performance of these models with TALiSMaN in simulated environment and compare them with TALiSMaN without traffic prediction model. Overall, weighted moving average showed promising results in reducing the packet dropped while capable of maintaining the usefulness of the streetlight when compared to TALiSMaN scheme, especially during rush hour.
\end{abstract}

Keywords-Traffic prediction; adaptive street lighting; smart cities; energy efficient; network congestion

\section{INTRODUCTION}

Street lighting is an essential public service that ensures the road safety for the public during the night [1]. The number of streetlights increased when an area is getting urbanise. Thus, the electricity and energy consumption of street lighting also increased linearly with streetlight. According to [2], this has various impacts such that it has significantly affected the cities' energy use and burden the municipal budgets.

With the notion of Networked Street Lighting, also known as intelligent streetlight system, street lighting can be operated autonomously by observe the changes in surroundings. [3] indicated that intelligent streetlight system can reduce high electricity and energy consumption of traditional streetlights, while ensuring public safety and economic growth.

One way to manage Networked Street Lighting is implementing the Wireless Sensor Networks (WSNs) onto the lamp pole. Application of WSN consists of issues where constant monitoring and manual resolving is impossible. WSN need to be able to adapt the real time network traffic to ensure quality of service and avoid malfunction.
There have been a few attempts on street lighting management, among many of them, distributed Traffic-Aware Street Lighting Scheme Management Network (TALiSMaN) [4] is one of the promising schemes for the adaptive streetlight system. As it is distributed, TALiSMaN performs dynamically and remotely without depending on human intervention. Based on [4], TALiSMaN was equipped with WSNs to improve the efficiency in power consumption and to facilitate network communication among the lamp pole effectively.

TALiSMaN could work well in urban area, but that is not the case for rural areas. Due to poor access to power grid, TALiSMaN-Green [5], which was an improved version of TALiSMaN scheme that made use of green energy to power the streetlights. TALiSMaN-Green aimed to further improve energy consumption efficiency, cost saving and less carbon footprint. TALiSMaN-Green adopted solar panels and battery [5], to ensure the power supplying is ample for the usage until the next daylight, and implements Artificial Intelligence (AI) to predict energy demand and allocate energy accordingly.

On the contrary, the urbanisation process and urban population expansion bring great pressure to the urban traffic management that result in traffic congestion [6]. This is a burden for both TALiSMaN and TALiSMaN-Green as the scheme requires real-time data to transfer among streetlights. Besides, road traffic congestion will lead to network overload, due to constant sensing and broadcasting in both schemes, which prone to network congestion, delay in data transmission, packet lost as well as wastage of energy.

In smart streetlight scheme, even a slight delay is unbearable as operation of networked streetlight is totally dependent on the information exchange between streetlights. Streetlight expects instant lights on before the commuters are approaching. A slight delay of the scheme could lead to streetlight unable to illuminate on time, thus reducing commuters' visibility and perception of safety when in a dark environment. This is crucial as it might affect the utility of TALiSMaN schemes and may cause more harm.

Moreover, when packet dropped increases, streetlight is unable to receive the packet to turn off the light, thus causing drainage of energy and result in performance degradation. The issue of energy wastage is affecting TALiSMaN-Green the most as it only possesses limited energy budget for each sensor node. 
Traffic forecasting is crucial for efficient development of road networks [7]. In this study, we enhance TALiSMaN with three moving average computation technique to ease the severity of packet drop and network congestion. The aim of this study is to use road traffic volume predictor to establish a stable connection and efficient throughput to ensure the attainability of streetlight throughout the entire night, thereby achieving streetlight usefulness from evening until the next morning. We evaluate the performance of proposed solution by comparing with the original TALiSMaN scheme using a linear streetlight topology. From the simulation results, the proposed solution reduces packet dropped and maintains the usefulness of TALiSMaN scheme throughout the whole night.

The rest of this paper proceeds with the background study of the previous work on intelligent streetlight and the issues occur. Followed by, the related works on prediction models. Next, a brief description on the main idea and structure of the proposed system. Then, evaluation of proposed system compared with TALiSMaN scheme. Finally, conclude the paper and highlights recommendation for future work.

\section{BACKGROUND}

\section{A. TALiSMaN and TALiSMaN-Green}

TALiSMaN is a traffic-aware lighting scheme management network that makes use of sensors for autonomous control of the brightness of each streetlight via a distributed network [4]. Instead of depending on a centralised operational control, TALiSMaN is designed to be adaptive to the different behaviours of road users and enable each streetlight to operate independently using a short-range mesh network.

According to [5], 150 meters is the range where optimum lighting condition in the surrounding of a pedestrian should have. For the purpose of efficient energy usage, the light intensity of the streetlight decreases gradually in the range of $150 \mathrm{~m}$ from every direction (distance between S1 to S5 and S5 to S9) when the sensors detect the presence of pedestrian progress further; while ensures ample of visibility in that environment, as shown in Fig. 1. As for motorists, streetlights within a $100 \mathrm{~m}$ radius from the detected vehicle are completely turned on, as presented in Fig. 2. Author in [5] claimed that TALiSMaN can reduce energy usage by $45-98 \%$ based traffic condition of the road.

In TALiSMaN, consistent delivery of data and network congestion control are necessary to achieve the full potential of TALiSMaN. Since the sensor in TALiSMaN scheme has no knowledge of the travel direction of detected users, all the streetlight will keep on broadcast data to each other under the predefined perimeter via a refined flooding protocol. In other words, if the road user is located at S5 in Fig. 1, the streetlight will start broadcasting data to S4 and S6. If S1 to S9 is within $150 \mathrm{~m}$ perimeter, then the broadcast will proceed from S4 to S3 and S5, S6 to S5 and S7, and continue the broadcasting until S9 to S8 and S1 to S2. The broadcast of data packet will repeat every 0.5 seconds and stop broadcasting after 15 seconds. When the road user approaching to S6, S5 will still broadcast the same data to other streetlight, and at the same time S6 also starts to broadcast the same data as S5, by means of informing other streetlight the distance of road user and to predefined light intensity based user's distance.

One of the setbacks of TALiSMaN is that it is vulnerable to data transmission delay. In the predefined area, it requires an instant end-to-end transmission to all the streetlights. Fig. 2 can further describe the important of the high precision on data transmission in TALiSMaN. Assume that the distance between streetlight in Fig. 2 is $30 \mathrm{~m}$ apart from each other, a car is travelling with a constant speed of $40 \mathrm{~km} / \mathrm{h}$ through a $120 \mathrm{~m}$ of street, TALiSMaN needs to ensure generated data packets takes about 2 seconds to broadcast across the street distance $(120 \mathrm{~m})$ [8]. The reason is to ensure the streetlights within $100 \mathrm{~m}$ surrounding the car is turned on when the car is approaching the next streetlight. When streetlight did not turn on before the car arrive, it will reduce the usefulness [5] of the streetlight.

There is another setback during heavy road traffic. During heavy traffic, the WSN prompts to be congested which causes packet loss at the rate of between $23 \%$ and $29 \%$. As discussed previously the data broadcast between streetlights is delay sensitive and it might cause TALiSMaN-Green fails to operate until the next day. This is because when sensor node is not receiving packet, the streetlight may remain switch on, which causes overburden of sensor node and resulting in rapid drainage of energy. If the scheme is unable to ensure instant transmission of data to the neighbouring streetlight, it will be dampening the potential and performance of this scheme, and hence resulting in streetlights slow to respond to the road users' movements and less efficiency of energy consumption.

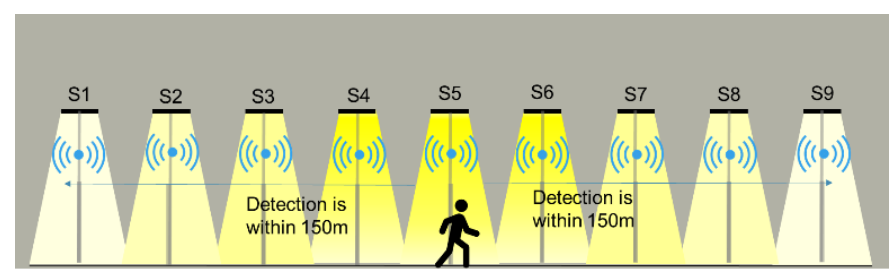

Fig. 1. TALiSMaN Street Lighting Distribution for Pedestrian Adopted from [5].

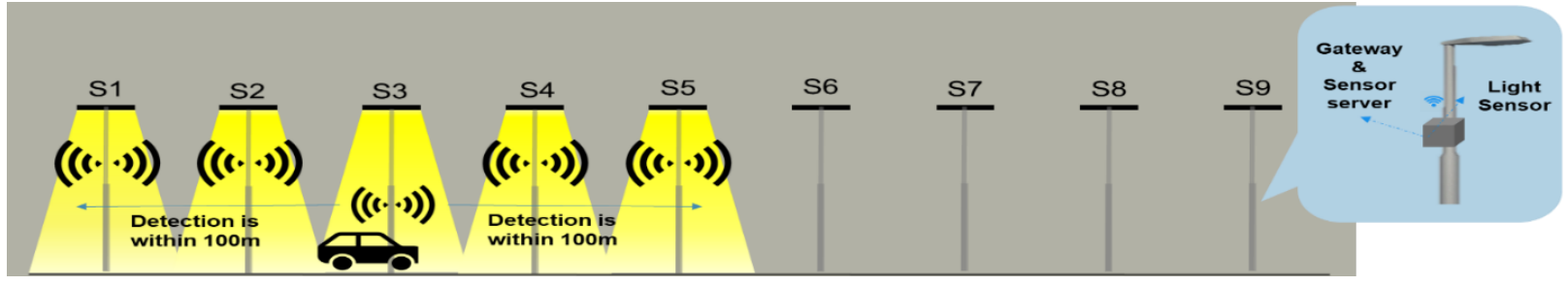

Fig. 2. Street Lighting Distributions for Motorist Adopted in TALiSMaN [4]. 


\section{B. Related Work on Prediction Model}

There have been some attempts on tackling the issue of network congestion, one of the methods is applying the prediction model to forecast the situation of transmission of data in real time of a network, then determine the action to best prevent the happening of network congestion. There comes another challenge in this part which is to perform model selection that best suited for the TALiSMaN scheme since there are so many kinds of the prediction model to opt for. Statistical model and Artificial Intelligence model for traffic forecasting are compared in [9]. This section discusses the various techniques or algorithms of predictions and their respecting use case.

Statistical model such as Auto Regressive Integrated Moving Average (ARIMA) has been quite popular around as a predictive model for various domains, especially transportation research in [10], [11] and [12]. Author in [13] realised the importance of high accuracy in traffic prediction for resource management in cloud computing. ARIMA was apt to predict short term traffic conditions, but in order to gain accurate results it required huge amount of historical data which is not ideal for a limited available memory on a WSN node.

Artificial Intelligent models such as Machine Learning and Deep Learning are some buzzwords these days. Machine Learning is a subset of artificial intelligence that can create and perform algorithm itself without human intervention [14]. Deep learning is a subset of Machine Learning that consist of numerous layers of algorithms, also known as artificial neural networks [15]. Author in [16] proposed a machine-learning technique, namely support vector machine (SVM) [17] to predict vehicle travel times. Author in [18] suggested a neural network incorporate with a fuzzy method to manage non-linear data in traffic data. In 2017, [19] adopted Long Short-Term Memory Neural Network (LSTM) for travel prediction by utilising temporal-spatial correlation techniques through a twodimensional network. The cost for training these models is computationally expensive, even though frequent updating is being prohibitive [20]. While these approaches can perform well, there is one drawback that is these models are technically difficult to implement on large scale networks.

ARIMA is more advanced version of Statistical Model, it combines the technique of Auto Regression and Moving Average. However, it is not a suitable solution in our study as the computing resource is limited for TALiSMaN-Green that operating with a WSN node. Thus, we argue that the weightage on deciding the prediction model should give to efficiencies over performance. Whereas Artificial Intelligence, other than heavy taxing on computing resource, it is far too complex to implement on TALiSMaN-Green. Thus, this study is more favourable on simpler approach for the prediction model.

\section{PROPOSED SOLUTION}

The proposed solution is illustrated in Fig. 3. The purpose of this scheme is to enhance the performance of TALiSMaN by reducing packet drop to ensure the accessibility of streetlight during heavy rush hour, so that streetlight can turn on when it is required. Furthermore, the proposed solution aims to maintain the usefulness of TALiSMaN even after adopting the proposed scheme, as to ensure it can perform in TALiSMaNGreen. Reason is that, with the reduction in duplicate packet broadcast, there are low probability of network congestion and exhaustion of sensor node, thus allow TALiSMaN-Green to reserve ample energy to operate during the next day while maintain the usefulness of TALiSMaN. As mentioned before, due to the challenges of network congestion in TALiSMaN and limited power budget in TALiSMaN-Green, a proper measure must be taken. The following subsections detail the proposed approach to meet these requirements.

Fig. 3 presents the overall idea of the proposed solution. LED is the core component of the TALiSMaN scheme as it is more energy efficient compare to compact fluorescent lamps (CFL) and Incandescent Light Bulbs [21]. It also provides better brightness and have a longer lifespan [22]. Under this scheme, each of the streetlight is powered by electrical grid to ensure sufficient and stable power supply [23], [24]. The backbone of this networked streetlight is powered by WSN, that enable transmission of data for different purposes, such as monitoring, adjusts brightness of LED or merely for notification purpose [4], [5]. TALiSMaN is embedded with AI capability that allows autonomously performance by responding to the type of road users and dynamically adjusts the lighting scheme based on users' proximity.

The prediction model technique (shown in blue colour box in Fig. 3) acts as a place holder that will swap in and out and to find out which prediction model will yield the best result possible. The prediction model consists of three phase, namely forecast phase, detection phase, control phase. This study proposes to use some simple short-term prediction models that are known for consuming less power to perform the computation of the prediction model. The prediction model is used to predict traffic for the next $t$ period and categorise the traffic into light or heavy traffic based on the historical traffic pattern, then control the packet propagation to maintain the usefulness of the existing scheme. When prediction shows the occurrence of heavy traffic during the next $t$ period, the proposed solution will signal the streetlight to remain turn on until the prediction shows light traffic. When prediction shows light traffic for the following $t$ period, the streetlight operation will proceed as predefined in TALiSMaN scheme. Fig. 4 illustrates the workflow of the proposed system.

\section{A. Traffic Prediction Model}

The moving average is a smoothing method that estimates future values that heavily depends on the historical data. This study will explore three moving average algorithms to plug and play with the TALiSMaN scheme to determine which is the most power efficient method and yet most effective in reducing the network congestion. These algorithms are Simple Moving Average, Exponential Moving Average and Weighted Moving Average.

Before delving into how these prediction methods implement into TALiSMaN, we need to determine three moving average parameters that include the traffic data at time period $t$, the lengths of the moving average periods $n$ and the type moving average method from those stated above. We apply root mean square error (RMSE) [25] to evaluate the accuracy of the method to predict road traffic data $x_{t}$. 


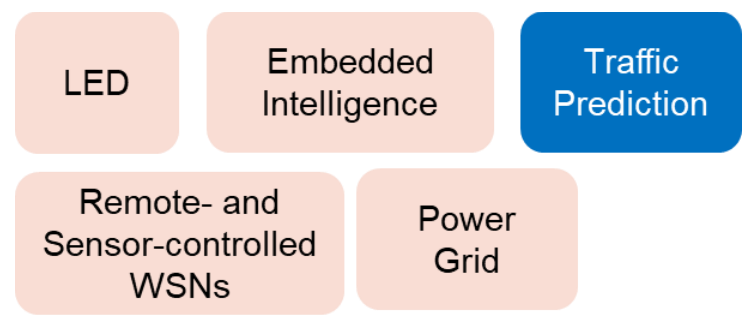

Fig. 3. Components of Refined TALiSMaN.

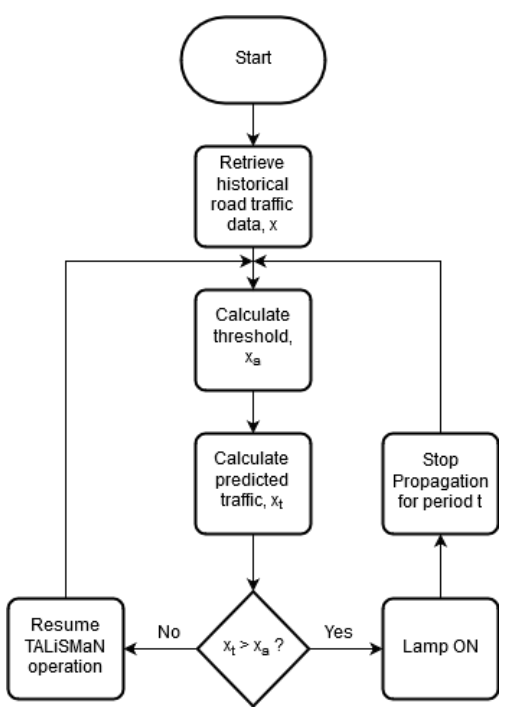

Fig. 4. Workflow of Proposed Syetem.

$\mathrm{RMSE}=\sqrt{\left(n^{-1} \sum_{i=1}^{n}\left(x_{f_{i}}-x_{t_{i}}\right)^{2}\right)}, n>i$

Where:

$x_{f_{i}}=$ forecasts traffic value at period $t$

$x_{t_{i}}=$ actual traffic value generated by TALSMaN scheme at period $t$

$n=$ number of periods

The following is an explanation of the steps to obtain those three parameters.

Step 1: (Generation of initial data): Historical traffic data is collected over a time interval from the simulation of TALiSMaN. The simulation is set to run from Monday until Sunday, where streetlights operate from 16:00 to 08:00 the next day, with 3508 [26] road users per day, over a period of 100 weeks. Three datasets are generated, where 10,000 sets of data are for training; 950 datasets each for testing and validation purpose. Note that there is no real data used as the simulated traffic pattern is based on the traffic distribution profile adopted from [27]. The generated road traffic data is adequate and equivalent to real life road traffic scenario, as the car is randomly injected at any point of the road, either moving to the left or right. The car is also randomly added at any time based on the traffic ratio predefined in the road distribution profile for that specific time period.
Step 2: (Aggregation of data); Simulation results are accumulated into weekly format. Since the road traffic profiles indicate perceptible trends throughout the days of week as shown in Fig. 5, thus it is realistic to assume historical traffic data from previous week of the same day and time can provide a more accurate prediction than previous day data. For instance, when predicting for time period 17:00 on Monday, assuming prediction length is two days of week, the prediction calculation should be the generated traffic data for time period 17:00 of Monday from two previous weeks.

Step 3: (Determination of t value): To produce an accurate traffic volume prediction, time period $t=\{24,288,1440\}$ is considered. The time period $t=\{24,288,1440\}$ represent onehour, five-minutes and one-minute time frame respectively. The equation to calculate the time period is shown below.

$\mathrm{t}=\frac{\text { tal hours in a day } * 3600 \mathrm{sec}}{\text { selected time period } * 60 \mathrm{sec}}$

Based on Fig. 6, $t=1440$ shows the most accurate prediction with the lowest error value, when evaluated with three of the prediction methods.

Step 4: (Determination of $n$ value): Since data aggregation is in weekly format, the moving average prediction length $n$ will represent the number of previous $n$ days. The $n$ value varies from 1 to 50 days, with $t=1440$ are evaluated with RMSE for the three prediction methods. From Fig. 7(a), (b) and (c), the RMSE values that has the lowest minimum values of each predictor is chosen as their respective $n$ values. Hence, $n=4$ is chosen for three of the predictors as they have the least minimum error.

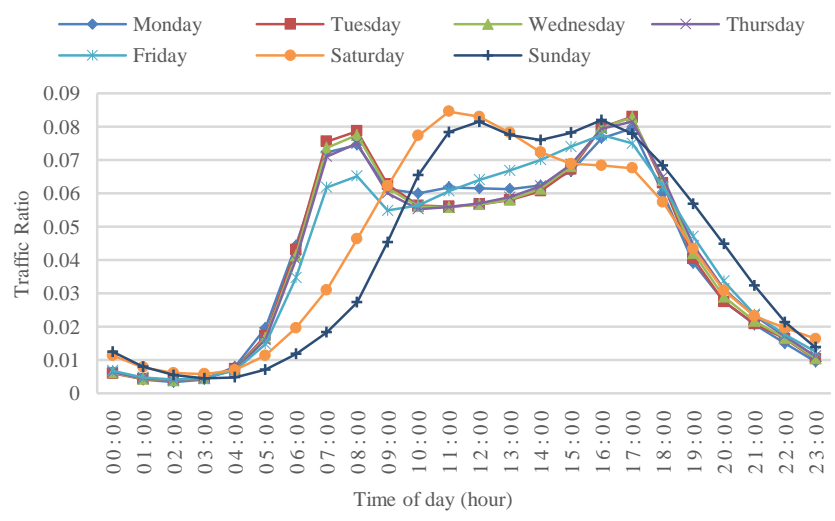

Fig. 5. Road Traffic Distribution Ratio throughout the Week based on [27].

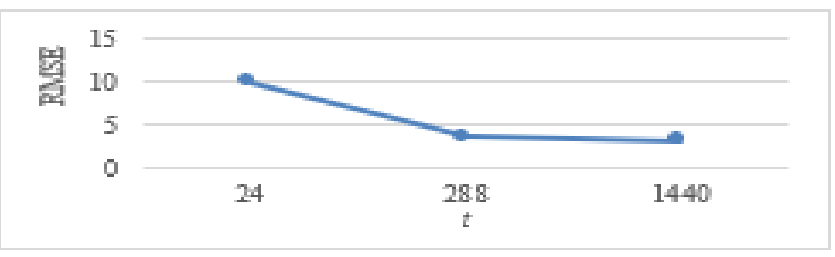

Fig. 6. Average Prediction Error of Predictors with $n=4$ Days Against $t$ Values. 


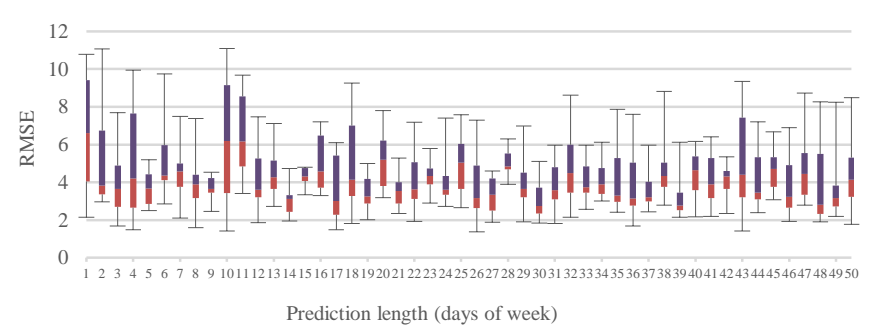

(a) SMA

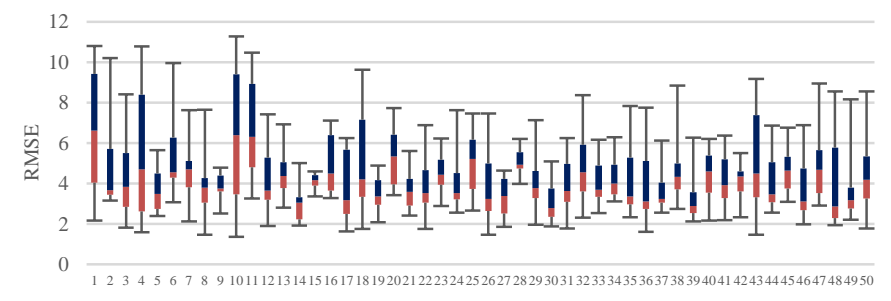

Prediction length (days of week)

(b) EMA

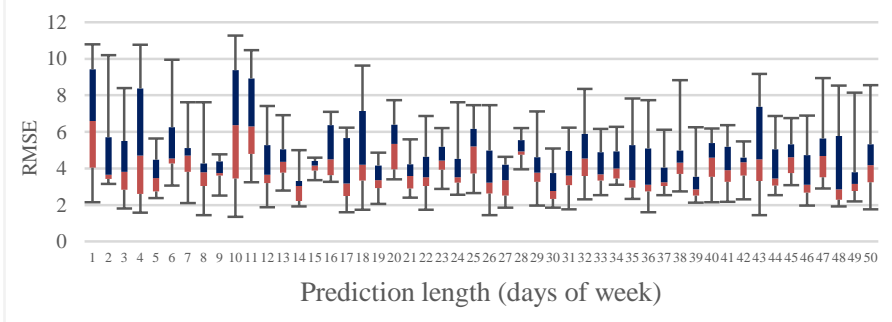

(c) WMA

Fig. 7. The Interquartile Ranges of RMSE Result for different Predictors at different Prediction Length, $n$ Values. The Error bars Indicate the Minimum and Maximum of MAE values.

Step 5: (Determination of prediction model): In this step, three of the prediction methods are simulated and tested with $t=1440$ and $n=4$ at traffic volume of 3508. To illustrate, the scheme initially divides the total time in a day into $t$ equal size timeslot that is in every one-minute traffic predictor predict traffic volume for the future timeslot (next one-minute) while collecting actual traffic one second after the previous timeslot. Thus, the calculation for each predictor is derived in the subsection below. Before delving into which prediction methods is more suitable to implement into TALiSMaN, some brief description is made on each of the algorithms.

1) Simple moving average: There are many variants of the moving average method, Simple Moving Average (SMA) as the name of method is the simplest method to construct among them. As shown in the formula (3), the calculation of SMA is by taking the average of traffic volume data $x_{t}$ at period $t$ for previous $n$ days of week. Since mentioned above $t=1440$ and $\mathrm{n}=4$, in order to calculate SMA at timeslot 196, the $\mathrm{x}_{196}$ from previous four days are used for obtaining the average value.

$S M A_{t}=\frac{x_{t}+x_{t-1}+\ldots+x_{t-(n-1)}}{n}$

$=n^{-1} \sum_{i=0}^{n-1}\left(x_{t-i}\right), n>i$
Where:

$x_{t}=$ road traffic volume at time period $t$

$n=$ number of days of week

2) Exponential moving average: The main difference between Exponential Moving Average (EMA) and SMA is the sensitivity towards variation in the most current data during calculation [28]. Based on the equation, the weighting factor decreases for older historical traffic data while provide more weightage towards recent traffic data to accurately predict the traffic trend. The accuracy of EMA relies on the smoothing factor of the recent data and the prediction length [29]. As when prediction length is longer, the lesser the weightage value causing it to be insignificance towards change.

$E M A_{t}=a * x_{t}+(1-a) * E M A_{t-1}$

Where:

$a=\frac{2}{1+n}[29]$ ?

$x_{t}=$ road traffic volume at time period $t$

$n=$ number of days of week

$E M A_{t-1}=$ Forecast of past time period road traffic volume

3) Weighted moving average: Like EMA, Weighted Moving Average (WMA) applies less weight on the past data while more on the current data. This is due to the calculation of multiplying each current value by a weighting factor as follows:

$W M A_{t}=\frac{\sum_{i=0}^{n-1}(n-i) x_{t-i}}{a}, n>i$

Where:

$a=n * \frac{1+n}{2}$

$x_{t}=$ road traffic volume at time period $t$

$n=$ number of days of week

\section{B. Congestion Detection based on Traffic Pattern}

Every sensor node will store up to a month of historical road traffic data and will undergo categorisation before proceeding with prediction process. The categorisation of predicted traffic data will utilise the road traffic threshold value as a metric to determine the congestion situation. The calculation for road traffic threshold value (assume is $x_{a}$ ) is compute using the average value of the road traffic data on a specific day from previous week.

For instance, the first step is determining the operating hours of streetlight in a day then perform data collection. Typically, the operation hour of a streetlight in a day starts from 12am until 8am, then $4 \mathrm{pm}$ until 11pm, as shown in Fig. 8 The traffic threshold value is obtained by taking the average value of the road traffic data in Fig. 8, where $x_{a}$ is 107 vehicles. After the prediction is performed, the prediction result at time period $t$ (assume is $x_{f}$ ) will be compared with the 
average value obtained previously (which is 107 as mentioned above). Predicted traffic data greater than the threshold value (i.e. $\left.x_{f}>107\right)$ is categorise as congested, while predicted data lesser than threshold value $\left(x_{f}<107\right)$ is categorise as noncongested period.

$x_{a}=\frac{\sum_{i=1}^{n} \mathrm{x}_{t_{i}}}{n}, n>i$

Where:

$x_{a}=$ road traffic threshold value for a day

$x_{t}=$ road traffic volume at time period $t$

$n=$ number of time periods

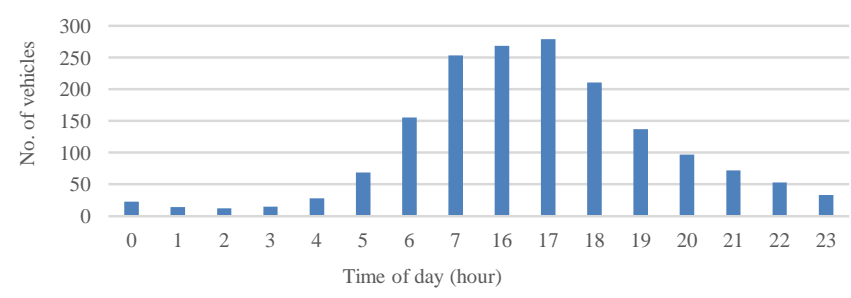

Fig. 8. The Simulation Results of Monday Vehicle Distribution Generated by StreetlightSim with 3508 Road users Per Day.

\section{Congestion Control Strategy}

The prediction models will be applied to TALiSMaN after obtaining prediction value to determine the action for the next period $t$ (i.e. one-minute). If the result of prediction $x_{t}$ indicates there is going to have a congestion for a certain amount of time $t$, then TALiSMaN will stop the broadcasting of data among streetlight, while the streetlight remains turned on at time $t$. To illustrate, if prediction results indicate there will be heavy traffic from $4.00 \mathrm{pm}$ to $4.05 \mathrm{pm}$, then TALiSMaN will stop the packet propagation of every sensor node and keep the streetlight on for one-minute. On the other hand, if there is no congestion, then packet propagation will proceed as intended in TALiSMaN.

\section{RESUlT ANALYSIS AND DISCUSSION}

This study proposed prediction model to determine the heavy traffic congestion period that affects the packet dropped rate, and provide control measures to reduce packet dropped, while ensure attainability of streetlight. By predicting the future traffic volume at time period $t$, it is possible to reduce packet dropped through traffic condition detector and packet propagation controller. The proposed scheme implemented with each of the prediction model is analysed and discussed based on the comparison with TALiSMaN. This section will also describe the simulation tools, configuration, assumptions and evaluation parameters.

\section{A. Simulation Setup}

The proposed scheme is implemented in StreetlightSim. StreetlightSim is an open-source simulation environment that is used to implement TALiSMaN as well as TALiSMaN-Green [26]. The simulation environment utilised both OMNeT++ and SUMO tools, to represent all the performance and operation of streetlights, such as traffic detection, traffic pattern generation, and evaluation of the practicality of the TALiSMaN and
TALiSMaN-Green schemes through the perspective of road user. The following parameters were used:

- Streetlights apply a linear streetlight topology in a residential area.

- The topology consists of 12 streetlights assign across the road of $360 \mathrm{~m}$ wide, shown in Fig. 9.

- Streetlights operate from 16:00 until the next day 08:00, to represent the streetlight operational hours in real life.

- The total road traffic is set to 3508 vehicles per day [26].

- The road traffic users will be added randomly into the simulation at any point of the road and anytme based on the traffic pattern predefined in the traffic distribution profile obtained from Southampton City Council [27].

- The simulation is run for 100 days.

- The $\mathrm{t}$ (time period) is set to 1440 , representing 1440 s (i.e. 1 minutes).

- The $\mathrm{n}$ (prediction length) is 4 days of week for every predictors.

\section{B. Evauation Parameters}

In this paper, the performance of proposed solution is evaluated in terms of Packet Dropped Ratio (PDR), Total Utility (TU) and Total Energy Consumption (TEC) as to demonstrate the effectiveness of real-time short-term traffic prediction in adaptive streetlighting.

PDR is chosen as the parameter as it shows the number of packets that are dropped during data transmission among streetlights. Furthermore, PDR can be used to identify problems that might lead to poor throughput or poor load distribution.

TU represents the measurement of the usefulness of streetlighting to road users obtained using utility model [29]. The usefulness model can be used as a parameter to determine the correctness, competence and impact of the proposed scheme towards existing scheme.

TEC refers to the total energy consumed by a streetlight in a day. As mentioned above, energy constraint is one of the factors affecting the performance of TALiSMaN that occurs when implement with the off-grid power supply. Since the battery used in off-grid scheme is very bulky and heavy to carry around, it is not feasible to constantly replace the batteries once it fails. It is crucial to extend the network lifetime as to ensure TALiSMaN scheme can perform effectively.

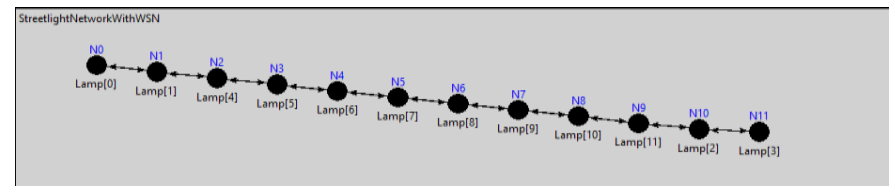

Fig. 9. A Linear Topology Set up with Twelve Streetlights. 


\section{Analysis and Discussion of Simulation Result}

Fig. 10 shows the interquartile ranges of packet dropped ratio for TALiSMaN with and without prediction generated throughout the night. The simulation results shown below incorporate with all the above-mentioned prediction parameters, which include the traffic volume of $3508, t$ value of 1440 and $n$ equals to 4 . Through observation, the SMA, EMA and WMA successfully reduce the packet drop of TALiSMaN scheme, with the range of $71.2 \%$ to $74.6 \%, 75.4 \%$ to $75.7 \%$ and $71.2 \%$ to $74.6 \%$ respectively. Without the traffic prediction, TALiSMaN has experienced a consistent packet dropping ratio, ranging between 636 to 680. Based on the simulation results, SMA and WMA have similar result, while EMA shows a slightly higher reduction in packet drop compare to SMA and WMA. This indicates that EMA is a better predictor in reducing packet dropped for TALiSMaN scheme.

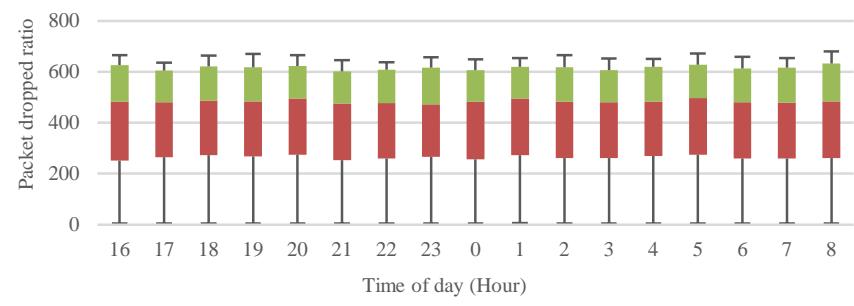

(a) TALiSMan without Traffic Prediction

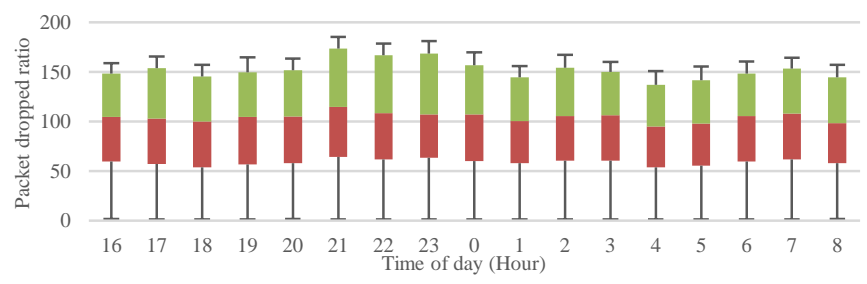

(b) SMA

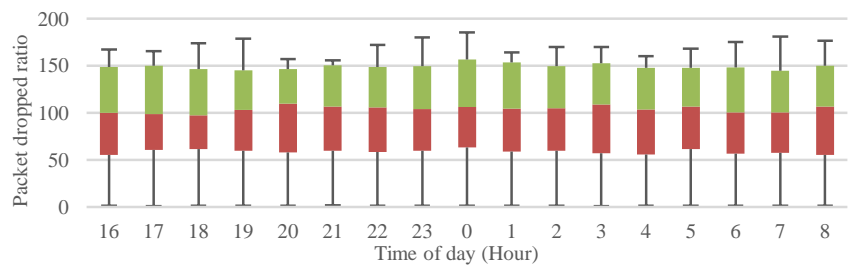

(c) EMA

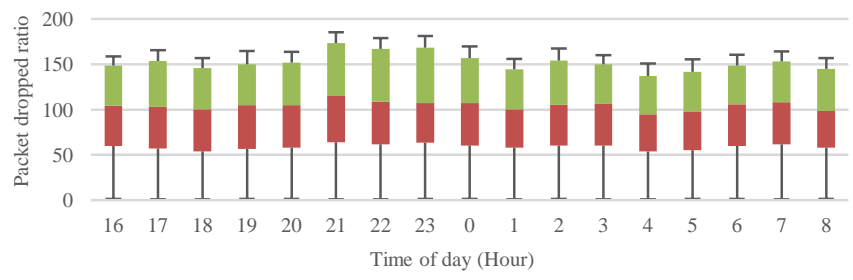

(d) WMA

Fig. 10. Average Packet Dropped Throughout the Operation Hour of the Streetlight between 16:00 to 08:00 the following Day. The Error bars Indicate the Minimum and Maximum Values of the Packet Dropped Ratio.

Fig. 11 shows the usefulness the application of the SMA, EMA and WMA in TALiSMaN scheme throughout the simulation days. It clearly shows that WMA provide consistent usefulness throughout the operation which is the highest among all scheme. Whereas SMA achieves the least usefulness, by dropping onto 0.6 on the 16th day and produce consistent usefulness throughout the operation days. TALiSMaN and EMA produce similar result throughout the simulation.

Fig. 12 illustrated the average energy usage in a day. Based on observation, all three predictors consume more energy than TALiSMaN because more energy is consumed to turn on the streetlight in full brightness when prediction indicates congested traffic condition. Overall, SMA consumed the most energy, whereas the energy consumption of EMA and WMA is nearly equal.

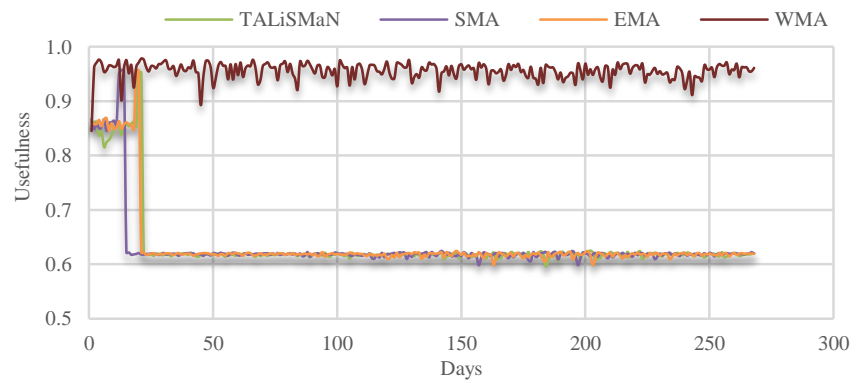

Fig. 11. Total usefulness Produced when Implemented with different Scheme at different Simulation Days.

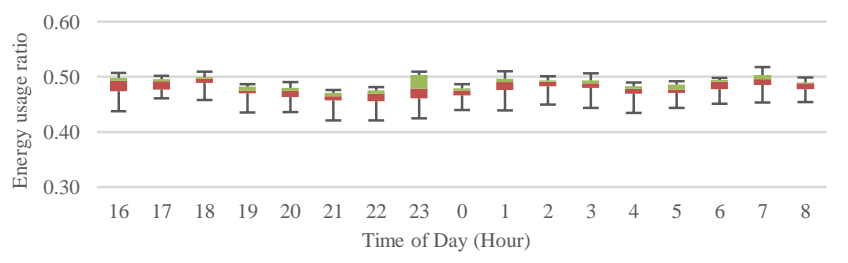

(a) SMA

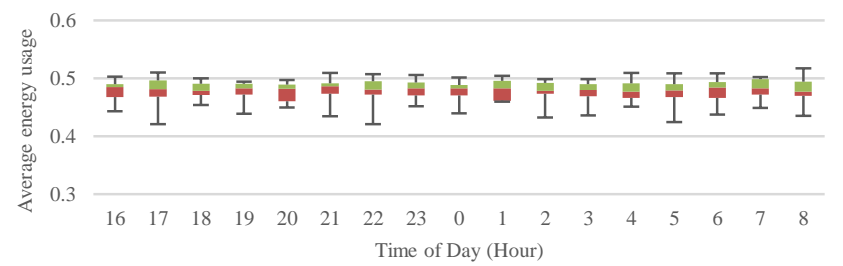

(b) EMA

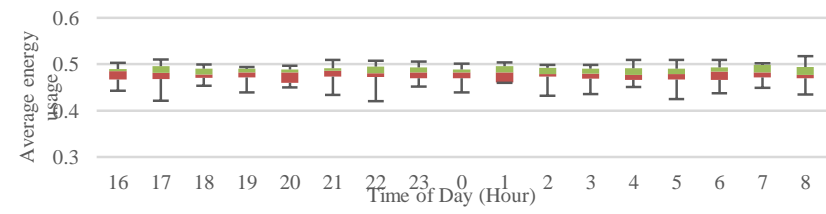

(c) WMA

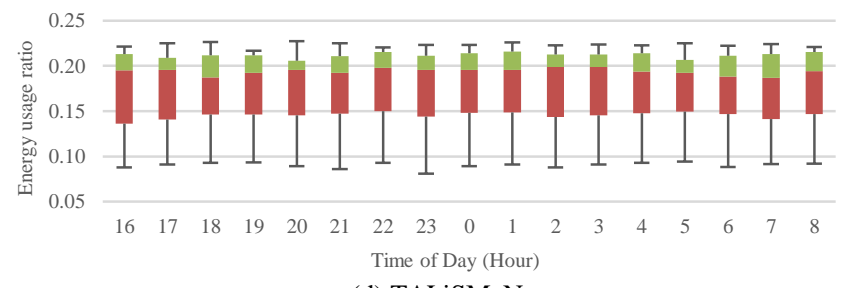

(d) TALiSMaN

Fig. 12. Average Energy usage Throughout the Operation Hour of Streetlight from 16:00 to 08:00 the following Day. The Error Bars Indicate the Minimum and Maximum Values of the Energy Consumed. 


\section{CONCLUSION}

The issue of network congestion in networked streetlighting system causes an increase in packet drop and network latency. Consequently, the streetlight is unable to utilise the full potential of TALiSMaN scheme if it fails or late to receive the signal to switch on or off. A streetlight is practically useless when it is unable to switch on in the dark. Contrary, streetlight continuously turn on throughout the night can cause excessive drainage of energy and prompt to operational failure. In order to tackle this issue in the networked streetlight system, a non-heavy computation prediction model to mitigate network congestion and packet dropped is adopted.

This study proposed and implemented three real-time shortterm predictions for TALiSMaN, an adaptive networked streetlighting system. These techniques utilise the historical traffic data, first to obtain the threshold value to detect the congestion condition, second, is to forecast periodic traffic volume. The forecast results are then used for signalling the streetlight to adjust the lighting, sensing and broadcasting operation to manage the congestion. The performance and analysis of TALiSMaN implemented with SMA, EMA, and WMA is measured and compared using packet dropped and energy efficiency.

\section{FUTURE WORK}

In the future work, it can be continuing with other more advanced short-term prediction method, potentially ARIMA, one thing to note that power consumption should keep in mind when selecting the method for future work. The performance of the scheme can be further enhanced by undergoing more tests on different structure of road and different time frame.

\section{REFERENCES}

[1] N. R. Velaga and A. Kumar, "Techno-economic evaluation of the feasibility of a smart street light system: a case study of rural India," Procedia-Social and Behavioral Sciences, vol. 62, pp. 1220-1224, 2012.

[2] S. A. E. Mohamed, "Smart street lighting control and monitoring system for electrical power saving by using VANET," International Journal of Communications, Network and System Sciences, vol.6,no.8,p.351, 2013.

[3] F. Marino, F. Leccese and S. Pizzuti, "Adaptive street lighting predictive control,” Energy Procedia, vol. 111, pp. 790-799, 2017.

[4] S. P. Lau, G. V. Merrett and N. M. White, "Energy-efficient street lighting through embedded adaptive intelligence," in 2013 International Conference on Advanced Logistics and Transport, 2013.

[5] S. P. Lau, A. S. Weddell, G. V. Merrett and N. M. White, "Energyneutral solar-powered street lighting with predictive and adaptive behaviour," in Proceedings of the 2nd International Workshop on Energy Neutral Sensing Systems, 2014.

[6] Z. Diao , D. Zhang, X. Wang, M. IEEE, K. Xie, S. He, X. Lu and Y. Li, "A Hybrid Model For Short-Term Traffic Volume Prediction In Massive Transportation Systems," IEEE Transactions on Intelligent Transportation Systems, vol. 20, no. 3, pp. 935 - 946, 2018.

[7] A. Singh, S. Ahamd, R. Singh, Ranjeet and R. Dubey, "Traffic Forecasting," International Journal of Scientific Research and Review , vol. 7, no. 3, pp. 1565-1568, 2019.

[8] S. P. Lau, A. S. Weddell, N. M. White and G. V. Merrett, "Poster: SolarPowered Adaptive Street Lighting Evaluated with Real Traffic and Sunlight Data," in Proceedings of the 13th ACM Conference on Embedded Networked Sensor Systems, 2015.

[9] M. G. Karlaftis and E. I. Vlahogianni, "Statistical methods versus neural networks in transportation research: Differences, similarities and some insights," Transportation Research Part C: Emerging Technologies, vol. 19, no. 3, pp. 387-399, 2011.
[10] K. Kumar, M. Parida and V. K. Katiyar, "Short term traffic flow prediction for a non urban highway using artificial neural network," Procedia-Social and Behavioral Sciences, vol. 104, pp. 755-764, 2013.

[11] A. Das, S. Barua and K. C. Roy, "Estimation of traffic arrival pattern at signalized intersection using ARIMA model," International Journal of Computer Applications, vol. 128, no. 1, pp. 1-6, 2015.

[12] B. Moghimi, A. Safikhani, C. Kamga and W. Hao, "Cycle-length prediction in actuated traffic-signal control using ARIMA model," Journal of Computing in Civil Engineering, vol. 32, no. 2, p. 04017083 , 2017.

[13] H. Mehdi, Z. Pooranian and P. G. Vinueza Naranjo, "Cloud traffic prediction based on fuzzy ARIMA model with low dependence on historical data," Transactions on Emerging Telecommunications Technologies, p. e3731, 2019.

[14] F. Jiang, Y. Jiang, H. Zhi, Y. Dong, H. Li, S. Ma, Y. Wang, Q. Dong, H. Shen and Y. Wang, "Artificial intelligence in healthcare: past, present and future," Stroke and vascular neurology, vol. 2, no. 4, pp. 230-243, 2017.

[15] A. B. Levine, C. Schlosser, J. Grewal, R. Coope, S. J. Jones and S. Yip, "Rise of the machines: Advances in deep learning for cancer diagnosis," Trends in cancer, vol. 5, no. 3, pp. 157-169, 2019.

[16] C. H. Wu, J. M. Ho and D. T. \& Lee, "Travel-time prediction with support vector regression," IEEE transactions on intelligent transportation systems, vol. 5, no. 4, pp. 276-281, 2004.

[17] N. G. Polson and S. L. Scott, "Data augmentation for support vector machines," Bayesian Analysis, vol. 6, no. 1, pp. 1-23, 2011.

[18] C. Quek, M. Pasquier and B. B. S. Lim, "POP-TRAFFIC: A novel fuzzy neural approach to road traffic analysis and prediction," IEEE transactions on intelligent transportation systems, vol. 7, no. 2, pp. 133146, 2006.

[19] Z. Zhao, W. Chen, X. Wu, P. C. Y. Chen and J. Liu, "LSTM network: a deep learning approach for short-term traffic forecast," IET Intelligent Transport Systems, vol. 11, no. 2, pp. 68-75, 2017.

[20] Y. Kamarianakis, W. Shen and L. Wynter, "Real-time road traffic forecasting using regime-switching space-time models and adaptive LASSO," Applied stochastic models in business and industry, vol. 28, no. 4, pp. 297-315, 2012.

[21] F. G. Montoya, A. Peña-García, A. Juaidi and F. Manzano-Agugliaro, "Indoor lighting techniques: An overview of evolution and new trends for energy saving," Energy and buildings, vol. 140, pp. 50-60, 2017.

[22] M. S. Islam, N. A. Chowdhury, A. K. Sakil, A. Khandakar, A. Iqbal and H. Abu-Rub, "Power quality effect of using incandescent, fluorescent, CFL and LED lamps on utility grid," in 2015 First Workshop on Smart Grid and Renewable Energy (SGRE), Doha, 2015.

[23] P. Nikolaidis and A. Poullikkas, "Cost metrics of electrical energy storage technologies in potential power system operations," Sustainable Energy Technologies and Assessments, vol. 25, pp. 43-59, 2018.

[24] P. T. Krein and M. A. Fasugba, "Vehicle-to-grid power system services with electric and plug-in vehicles based on flexibility in unidirectional charging," CES Transactions on Electrical Machines and Systems, vol. 1, no. 1, pp. 26-36, 2017.

[25] T. Chai and R. R. Draxler, "Root mean square error (RMSE) or mean absolute error (MAE)?-Arguments against avoiding RMSE in the literature," Geoscientific model development, vol. 7, no. 3, pp. 12471250, 2014.

[26] S. P. Lau, G. V. Merrett, A. S. Weddell and N. M. White, "StreetlightSim: A simulation environment to evaluate networked and adaptive street lighting.," in 2014 IEEE Asia Pacific Conference on Wireless and Mobile, 2014.

[27] European Platform on Mobility Management, "TEMS - The EPOMM Modal Split Tool," [Online]. Available: http://www.epomm.eu/tems. [Accessed 4 July 2013].

[28] J. Raiyn and T. Toledo, "Real-time short-term forecasting based on information management," Journal of Transportation Technologies, vol. 4, no. 1, p. 11, 2014.

[29] "Moving Averages - Simple and Exponential," [Online]. Available: https://school.stockcharts.com/doku.php?id=technical_indicators:movin g_averages. [Accessed 2312 2019]. 